\title{
Effect of doping on the magnetic ordering of quasi-one-dimensional antiferromagnets
}

\author{
A.A. Zvyagin \\ B. Verkin Institute for Low Temperature Physics and Engineering \\ of the National Academy of Sciences of Ukraine, 47 Lenin Ave., Kharkov 61103, Ukraine \\ E-mail: zvyagin@ilt.kharkov.ua
}

Max-Planck-Institut für Physik Komplexer Systeme, Nöthnitzer Str., 38, D-01187, Dresden, Germany

Received July 12, 2005

\begin{abstract}
We have studied theoretically how nonmagnetic dopants, which change the local coupling of spins to the host, affect the low-temperature thermodynamic characteristics of quasi-one-dimensional (Q1D) quantum spin antiferromagnets. Our theory qualitatively and, in some cases, quantitatively describe the behavior of the magnetic susceptibility and specific heat of the Q1D system $\mathrm{BaCu}_{2}\left(\mathrm{Si}_{1-x} \mathrm{Ge}_{x}\right)_{2} \mathrm{O}_{7}$. We have shown that in some cases the strong disorder in the distribution of characteristics of magnetic impurities in quantum antiferromagnetic spin chains can be the cause of magnetic ordering, if such chains are weakly coupled to each other, while for homogeneous chains and chains with a weak disorder a small enough coupling between chains does not produce magnetic ordering. For other values of the parameters, magnetic impurities can decrease the Néel temperature compared to that of the homogeneous Q1D spin system.
\end{abstract}

PACS: 75.10.-b, 75.10.Pq, 75.10.Nr, 75.40.-s

Keywords: Q1D quantum spin systems, magnetic impurities, ordering temperature for magnetic systems with impurities

In recent years interest in quasi-one-dimensional (Q1D) antiferromagnetic quantum systems has grown considerably. These systems are interesting from the theoretical viewpoint, because one can check the predictions of exact theories by comparing them with experimental data. On the other hand, many Q1D compounds are known to belong to the class of copper oxides, the study of which is important in the connection with the high- $T_{c}$ superconductivity. However, most of the results of theories and experiments in this field have been pertained to homogeneous spin chains. At least for the theory, that is clearly because the study of strongly correlated electron systems with disordered parameters is probably the most difficult problem of quantum condensed matter physics. Experiments on Q1D quantum spin systems with disorder have been known since the 1970s; see, e.g., [1-3]. First the renormalization group approximate theories were used to describe them; see, e.g., [4]. Much later, exact Bethe ansatz theories were constructed [5-8], which have shown that the finite concentration of magnetic impurities in quantum spin chains fundamentally change the behavior of the low-temperature characteristics of those chains. Recently, the class of solid solutions of copper oxides $\mathrm{BaCu}_{2}\left(\mathrm{Si}_{1-x} \mathrm{Ge}_{x}\right)_{2} \mathrm{O}_{7}$, which presents an almost ideal realization of quantum Q1D spin systems with disorder, was studied experimentally $[9,10]$. The goal of our work is to study how the concentration of nonmagnetic dopants (which, probably, introduce randomness in the distribution of exchange constants between magnetic ions [9] in such systems) affect the low-temperature properties of Q1D quantum spin antiferromagnets and to compare our results with experiments.

We start with the consideration of the Hubbard-like model of electrons on a one-dimensional lattice with a random hopping of electrons between sites of the lattice due to dopants, the Hamiltonian of which has the form

$$
\mathcal{H}=\sum_{j, j^{\prime}, \sigma} t\left(j, j^{\prime}\right) c_{j, \sigma^{\prime}}^{\dagger} c_{j^{\prime}, \sigma}+\sum_{j}\left(\frac{U}{2} n_{j, \sigma} n_{j,-\sigma}+\varepsilon_{j} n_{j, \sigma}\right)
$$


where the sums are over random positions of electrons, $c_{j, \sigma}^{\dagger}\left(c_{j, \sigma}\right)$ creates (annihilates) an electron with spin $\sigma$ at site $j, n_{j, \sigma}=c_{j, \sigma}^{\dagger} c_{j, \sigma}, t\left(j, j^{\prime}\right)$ are hopping elements, $U$ is determined by the Coulomb repulsion of electrons localized at the same site, and $\varepsilon_{j}$ are the energies of localized at sites $j$ electrons. The hopping integrals can be approximated [11] as over lap integrals

$$
t\left(j, j^{\prime}\right)=E_{0} \mathrm{e}^{-r_{j, j^{\prime}} / a}\left(1+\frac{r_{j, j^{\prime}}}{a}+\ldots\right),
$$

where $a$ is the Bohr radius and $E_{0} \sim U$ is the effective binding energy of the localized electron. The hopping integrals are random because of the disorder of the distribution of dopants. Here we limit ourselves to consideration of the case in which the localized electrons are in the magnetic state, i.e., the valences of the sites are close to 1 , because we are interested in the behavior of a spin chain with disorder. This condition is satisfied if $t^{2}\left(j, j^{\prime}\right) \rho\left(E_{F}\right) \ll-\varepsilon_{j}, U+\varepsilon_{j}$ [12]. Here $\rho\left(E_{F}\right)$ is the density of states at the Fermi level. Our analysis is close to Ref. 11 and we briefly repeat the main steps from it. It is natural to assume that the density of the localized magnetic moments $n_{l}$ depends on the density of dopants $c$ as $n_{l}=$ $=c \exp \left(-c / c_{\max }\right)$, where $c_{\max }$ is related to the critical distance between localization centers $r_{c}$ via $c_{\max }=1 / r_{c}$ for the $1 \mathrm{D}$ case. Then we can get the probability $P(r)$ to find the neighboring localized magnetic moment of a given site at distance $r$. The density of the localized magnetic moments can be written as $n_{l}=\int_{r_{c}}^{\infty} P(r) d r$, from which we obtain $P(r)=c \exp (-c r)^{c} . n_{l}$ has a maximum at $c_{\max }$, which, in fact, justifies our above assumption. The dependence of hopping integrals $t\left(j, j^{\prime}\right)$ (in what follows we shall denote those integrals simply as $t$ ), Eq. (2), implies $r(t) \approx a \ln \left(2 E_{0} / t\right)$. Hence, we obtain $\tilde{P}(t)=-(a c / t)\left(t / E_{0}\right)^{c a}$. The Kondo temperature of each localized magnetic moment can be written as [12]

$$
\begin{aligned}
& T_{K}=\sqrt{\frac{2 U \rho\left(E_{F}\right)}{\pi}} t \exp \left(-\frac{\left|\varepsilon_{j}\right|\left(\varepsilon_{j}+U\right)}{2 U_{\rho}\left(E_{F}\right) t^{2}}\right) \approx \\
& \approx D \exp \left(-\frac{|\varepsilon|(\varepsilon+U)}{2 U_{\rho}\left(E_{F}\right) t^{2}}\right) .
\end{aligned}
$$

Homogeneous distributions of local potentials $\varepsilon_{j}=\varepsilon$ are supposed, and the low-energy cut-off $D$ is used, as usual for the Kondo problem [12]. Defining $x=\ln \left(D / T_{K}\right)$ and $A=8 U \rho\left(E_{F}\right) E_{0}^{2} /|\varepsilon|(\varepsilon+U) \equiv$ $\equiv J_{\text {eff }} \rho\left(E_{F}\right)$, where $J_{\text {eff }}$ is the effective minimal exchange coupling of the magnetic moment of the localized electron to the chain, we obtain the distribution of local Kondo temperatures in the chain of correlated electrons

$$
\bar{P}\left(T_{K}\right)=\frac{c}{2 c_{\max } T_{K} x}(A x)^{-\left(c / 2 c_{\max }\right)} .
$$

The divergence of $\bar{P}\left(T_{K}\right)$ as $T_{K} \rightarrow 0$ (due to the factor $\left.T_{\underline{K}}^{-1}\right)$ is weakened by logarithmic factors. This is why $\bar{P}\left(T_{K}\right)$ can be normalized to unity over some interval $T_{\min } \leq T_{K} \leq T_{\max }$, where $T_{\max }$ is determined as $T_{\max }=D \exp (-1 / A)$. The distribution of local Kondo temperatures depends on $\varepsilon$, Coulomb repulsion $U$, density of states of electrons at the Fermi level, Bohr radius, and the density of dopants. However, Eq. (4) implies that such a set of parameters is realized in two main governing parameters: $c / 2 c_{\max }=n$ and $A$ (or $T_{\max }$ ). It is easy to see that for large enough domains of $T_{K}$ the distribution $\bar{P}\left(T_{K}\right)$ is reminiscent of $T_{K}^{\lambda-1}(\lambda<1)$, see Fig. 1. Such a distribution was used in $[1,6,8]$ without derivation. Notice that the energy parameter $G$ of $[6,8]$, where we used the distribution function $P_{\text {app }}\left(T_{K}\right) \sim T_{K}^{\lambda-1} / G^{\lambda}$, valid till $T_{K}=G$, can be related to $A$ (or to $T_{\max }$ ) via $G=$ $=D \exp \left(-n^{1 /(n+1)} / A^{n /(n+1)}\right)$.

For a quantum antiferromagnetic spin- $1 / 2$ Heisenberg chain it is natural to connect the local Kondo temperature with the effective coupling of the magnetic impurity to the chain $J_{i}$, by which the impurity is distinguished from other spins of the chain; cf. $[6,8]$. We shall study the case $0 \leq J_{i} \leq J$, where $J$ is the value of the exchange interaction between neighboring spins in the homogeneous Heisenberg

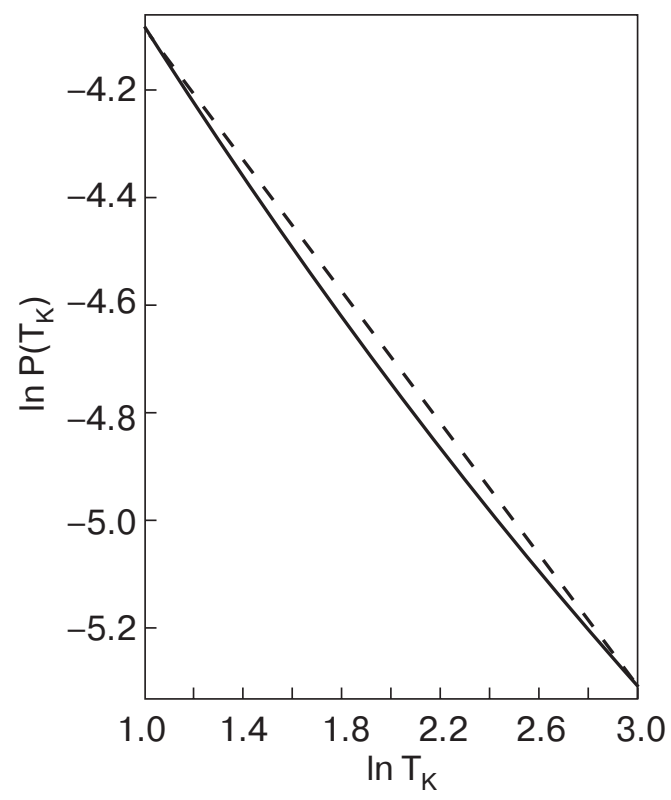

Fig. 1. The logarithms of the distribution function Eq. (4), the solid line, and $P_{\mathrm{app}}\left(T_{K}\right)=\mathrm{const} / T_{K}^{1-\lambda}$, the dashed line, as functions of $\ln T_{K}$ for $\lambda=0.39, A=1$, $n=0.5$, and $D=v_{F}=377 \mathrm{~K}$ (see text). 
chain. Then the local Kondo temperature of such an impurity in the chain is equal to $T_{K}=v_{F} \times$ $\times \exp \left(-\pi \sqrt{\left(J-J_{i}\right) / J_{i}}\right)$, where $v_{F}$ is the Fermi velocity of spinons in the homogeneous spin chain (notice that we use units in which $v_{F}$ has dimensions of energy, as usual for studies of such systems); cf. [6,8]. In the absence of the magnetic field we have $v_{F}=\pi J / 2$. It is natural then to use $D=v_{F}[6,8]$, so that for $J_{i}=J$ one has $T_{K}=v_{F}$. For the case $J_{i}=0$, i.e., if the impurity is totally decoupled from the chain, we have $T_{K}=0$.

With the obtained distribution function of local Kondo temperatures for a one-dimensional correlated electron system with electrons in the magnetic state it is easy to get average characteristics of the disordered Heisenberg spin chain using exact results for a single magnetic impurity in a Heisenberg chain; see, e.g., [8]. The low-temperature behavior of average values of the magnetic susceptibility, $\chi$ and the Sommerfeld coefficient of the specific heat, $c / T$ are equal to

$$
\langle\chi\rangle \propto \frac{\langle c\rangle}{T} \sim \frac{T^{\lambda-1}}{G^{\lambda}} .
$$

The ground state average magnetization can be calculated as $\left\langle M^{z}(T=0)\right\rangle \sim(H / G)^{\lambda}$. These weak powerlaw dependencies are related to Griffiths singularities [13] near the critical point $T=0$. The Wilson ratio at $T=0$ for the obtained distribution is equal to $2 \pi^{2} / 3$, characteristic for a Fermi liquid situation.

Now we can compare our theoretical results with the data of the experiments on $\mathrm{BaCu}_{2}\left(\mathrm{Si}_{1-x} \mathrm{Ge}_{x}\right)_{2} \mathrm{O}_{7}$ $[9,10]$. The parameters $D, \varepsilon, U$, and $E_{0}$, appropriate for that disordered correlated electron compound produce $A \approx 1$. It was shown that for $x=0.5$ the lowtemperature behavior of the magnetic susceptibility of that compound well reproduce the relation $\langle\chi\rangle \sim T^{-1}\left[\ln \left(\Omega_{0} / T\right)\right]^{-2}[4]$ for $\Omega_{0}=430 \mathrm{~K}$; see Fig. 1 of [9]. In Fig. 2 we plotted this dependence and the approximate one, which follows from our theory (the $\log -\log$ plot, as in Fig. 1 of [9]).

The results agree reasonably well for $\lambda=0.39$ (here we used $J=240 \mathrm{~K}$ of the case $x=0$ [9], which corresponds to $v_{F} \approx 377 \mathrm{~K}$ ). It is easy to check that for $\lambda=0.39, A=1$ and such a value for $v_{F}$, the distributions agree for the value $n=0.5$ (see Fig. 1). It correlates well with the concentration of Ge, $x=0.5$ of the experiment [9]. For these values of parameters we have $G \approx 201 \mathrm{~K}$, i.e., our distribution is valid well above the upper limit of temperatures, considered in Fig. 1 of [9]. Now, let us see how the exponent $\lambda$ is related to the concentration of dopants, $c$ (and, hence, to $n$ ). We checked that for $A=1$, and the same as above value of $v_{F}$, the case $\lambda=0.2$ of the approximate distribution reproduces well the obtained distribution

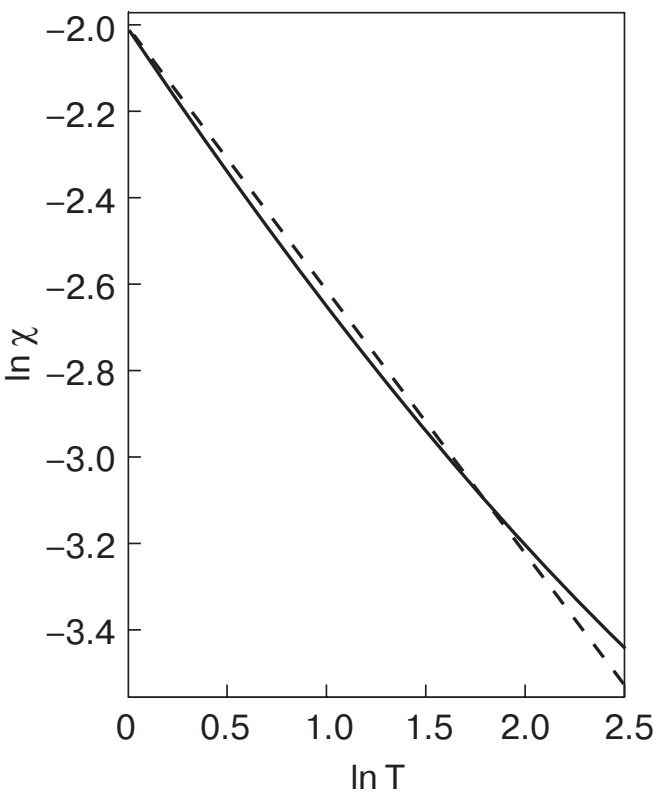

Fig. 2. The dependencies of the logarithm of the average magnetic susceptibility for the disordered spin-1/2 Heisenberg antiferromagnetic chain versus $\ln T$, calculated for two distribution functions: $\langle\chi\rangle \sim T^{-1}\left[\ln \left(\Omega_{0} / T\right)\right]^{-2}$ for $\Omega_{0}=430 \mathrm{~K}[4,9]$ (solid line), and $\langle\chi\rangle \sim T^{1-\lambda}$ for $\lambda=0.39$ (dashed line).

of Kondo temperatures, Eq. (4), for $n=0.005$. [It turns out that $\lambda \approx 0.2$ was observed in the experiments [2], but it is necessary to renormalize the values of $A$ and $v_{F}$ for them.] $\lambda=0.25$ (cf. [10]) is related to the value of $c=0.1 c_{\max }$, and $\lambda=0.3$ corresponds to $n=0.1$, i.e., the increase of the concentration of dopants produces the increase of $\lambda$. It correlates with the low-temperature behavior of the magnetic susceptibility and the Sommerfeld coefficient of the specific heat of $\mathrm{BaCu}_{2}\left(\mathrm{Si}_{1-x} \mathrm{Ge}_{x}\right)_{2} \mathrm{O}_{7}$ [10]. Notice that the behavior of those compounds for $x>0.5$ is probably connected with the renormalization of not only $n$, but also $v_{F}$ and $A$ (the doping in this case should be considered from the starting compound $\mathrm{BaCu}_{2} \mathrm{Ge}_{2} \mathrm{O}_{7}$, for which $J=500 \mathrm{~K}[9,10])$. We emphasize that renormalization group-based theories like [4] do not give the explicit dependence of the critical behavior of thermodynamic characteristics of disordered quantum spin chains, while our theory produces results that agree qualitatively (and even quantitatively for $x=0.5$ ) with the behavior of the disordered spin- $1 / 2$ compound $\mathrm{BaCu}_{2}\left(\mathrm{Si}_{1-x} \mathrm{Ge}_{x}\right)_{2} \mathrm{O}_{7}$. Similar results can be obtained for the correlation length $[6,8]:\langle\xi\rangle \sim$ $\sim(G / T)^{\lambda} / \pi \Delta+\ldots$, where $\Delta$ is the conformal dimension of the critical spin chain $\left(\Delta=(1 / 2)\left[(\Delta M)^{2}+\right.\right.$ $\left.+(\Delta D)^{2}\right]+n_{\mathrm{ph}}$, where $\Delta M, \Delta D$, and $n_{\mathrm{ph}}$ are integers or half-integers determining the spin projection of the chain, its momentum, and the number of particle-hole excitations, respectively). 
These results can be checked experimentally for $\mathrm{BaCu}_{2}\left(\mathrm{Si}_{1-x} \mathrm{Ge}_{x}\right)_{2} \mathrm{O}_{7}$ using the low-temperature inelastic neutron scattering.

Now, let us consider the behavior of Q1D quantum spin systems (i.e., with the interaction between chains being smaller than the coupling of spins along chains) with and without disorder. The magnetic susceptibility $\chi_{q}$ of a $\mathrm{Q} 1 \mathrm{D}$ quantum spin system, consisting of spin chains, weakly coupled to each other by the weak exchange interaction $J^{\prime}$ in the random phase approximation reads

$$
\chi_{q}(k, T)=\frac{\chi_{1}(k, T)}{1-z\left|J^{\prime}\right| \chi_{1}(k, T)},
$$

where $\chi_{1}(k, T)$ is the susceptibility of each chain as a function of the wave vector $k$ and temperature and $z$ is the coordination number. If the denominator of Eq. (6) becomes zero, a phase transition to a magnetically ordered state takes place. The temperature of magnetic ordering $T_{c}$ is determined from the condition

$$
\chi_{1}\left(T_{c}\right)=1 / z\left|J^{\prime}\right| .
$$

Notice that such a definition of the temperature of magnetic ordering in quasi-one-dimensional quantum spin systems has been used succesfully not only qualitatively but also quantitatively in many theoretical and experimental studies; see, e.g., [14,15]. Here one has to take the highest value of the magnetic susceptibility of the spin chain among those with different values of $k$. Consider first a ferromagnetic interaction between chains $J^{\prime}<0$, which corresponds to the case $k=0$. Here we closely follow the ideas recently set forth in [14]. Such an interaction produces an antiferromagnetic ordering in the total system with magnetic moments alternating in the direction of chains [15]. For the homogeneous spin chain $\chi_{1}(k=0)$ is less than some maximum value. For example, for a spin-1 $/ 2$ Heisenberg antiferromagnetic chain $\chi_{1 \text { max }}(k=0) \approx 0.14 / J, \quad$ cf. $[5,7,16]$. Hence, for small enough values of the inter-chain interaction, $\left|J^{\prime}\right|<\chi_{1 \max }(k=0) / z$, there are no solutions of Eq. (7), i.e., there is no magnetic ordering in such systems. For example, for a spin-1/2 Heisenberg Q1D system there is no such ordering for $\left|J^{\prime}\right|<0.14 J / z$ (we use units in which the Bohr magneton and $g$-factor are $\left.g \mu_{B}=1\right)$. Consider now spin chains with magnetic impurities (let us limit ourselves with the most interesting case, in which there is no spontaneous magnetization in each chain, even in the ground state). Then, according to $[5,7,8]$, magnetic susceptibilities of each chain with single impurities are less than their maximal values, which are determined by the Kondo temperatures, $\chi_{1 \max }(k=0) \sim$ const $/ T_{K}$. In that case, if $\left|J^{\prime}\right|<T_{K} /$ const $z$, Eq. (7) has also no solutions, and such a Q1D system is magnetically disordered. The same is true for spin chains with a weak disorder in the distribution of local Kondo temperatures of impurities (here by a weak disorder we mean narrow distributions with the values of the functions at their maxima being much larger than the values on their «tails»). For this case there is a maximum value of $\chi_{1}(k=0)$ also, and Eq. (7) has no solutions for small $\left|J^{\prime}\right|$. On the contrary, for chains with a strong disorder in distributions of local Kondo temperatures any, even a very weak coupling between chains $\left|J^{\prime}\right|$ produces magnetic ordering. The illustration of this consideration is depicted in Fig. 3.

Hence, one is faced with the interesting situation: Magnetic ordering is caused by a disorder. For the Q1D system consisting of weakly coupled spin-1/2 antiferromagnetic chains with the strong disorder considered above we have $\chi_{1}(k=0, T) \sim 1 / G^{\lambda} T^{1-\lambda}$. Then the critical temperature can be evaluated as

$$
T_{c} \sim\left(\frac{z\left|J^{\prime}\right|}{G^{\lambda}}\right)^{1 /(1-\lambda)} .
$$

How do impurities affect the critical temperature for $J^{\prime}>0$ ? This case produces alternating magnetic moments in all directions [15], and it is necessary to consider the staggered magnetic susceptibility of antiferromagnetic chains, $\chi_{1}(k=\pi, T)$. Unfortunately, this situation cannot be studied in detail, as for $J^{\prime}<0$. Only few results are known for the temperature behavior of the staggered magnetic susceptibility. However, for the most important case of the spin-1/2

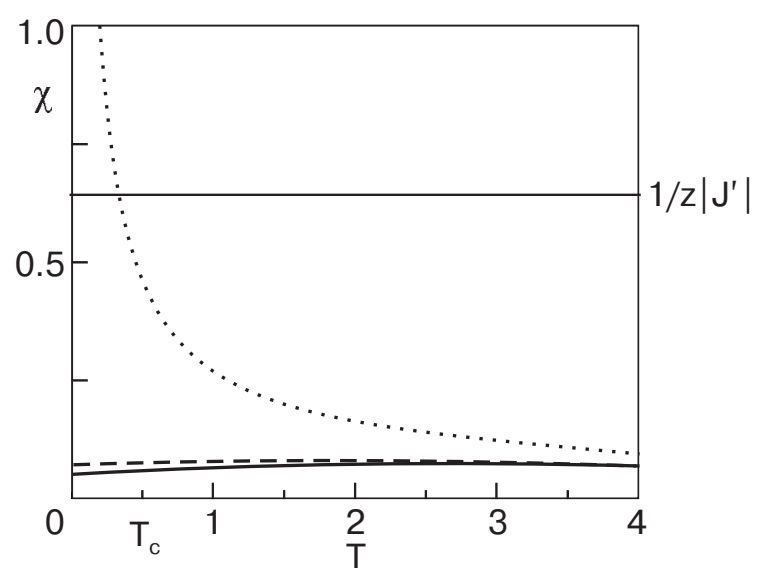

Fig. 3. Graphical solution of Eq. (6). The solid curve describes the temperature dependence of the homogeneous magnetic susceptibility of a homogeneous spin-1/2 Heisenberg antiferromagnetic chain; the dashed curve describes a similar inhomogeneous chain with weak disorder in the distribution of parameters of impurities; the dotted curve describes a similar inhomogeneous chain with strong disorder. 
Heisenberg antiferromagnetic chain we know that in the bosonization approximation $\chi_{1}(k=\pi, T) \approx$ $\approx 0.28 \sqrt{\ln (J / T)} / T$ [17]. Then the critical temperature is equal to $\left.T_{N} \approx 0.28 z J^{\prime} \sqrt{\ln \left(|J| / 0.28 z J^{\prime}\right)}\right) . T_{N}$ can be larger than the impurity-renormalized critical temperature. Nevertheless, generally speaking, for some range of parameters $T_{c}$ can be larger for a system with disordered magnetic impurities than in the case of weakly coupled homogeneous antiferromagnetic chains. On the other hand, for different sets of parameters the disorder in the distribution of parameters of interactions between spins in spin chains can cause a substantial reduction of the Néel temperature.

Let us consider the quasi-one-dimensional system $\mathrm{BaCu}_{2}\left(\mathrm{Si}_{1-x} \mathrm{Ge}_{x}\right)_{2} \mathrm{O}_{7}$. There is a weak coupling between chains. For $x=0$ the Néel ordering takes place at $T_{N}=9.2 \mathrm{~K}[9,10]$. We can estimate the value of the inter-chain interaction as $J^{\prime} \approx T_{N} / 0.28 z \sim 8.21 \mathrm{~K}$ (taking into account logarithmic corrections we have $\left.J^{\prime} \approx T_{N} / 0.28 z \sqrt{\ln \left(J / T_{N}\right)} \sim 4.55 \mathrm{~K}\right)$. For rough estimations we can suppose that the inter-chain interaction does not depend on dopants, and that we can use Eq. (8) for the case $J^{\prime}>0$ replacing $\left|J^{\prime}\right| \rightarrow J^{\prime}$. Then estimations for the critical temperature of the ordering for $\chi(T=0) \sim 0.01$ yield $T_{c} \sim 0.06 \mathrm{~K}$, i.e., $T_{c} \ll T_{N}$. Hence, in the case of $\mathrm{BaCu}_{2}\left(\mathrm{Si}_{1-x} \mathrm{Ge}_{x}\right)_{2} \mathrm{O}_{7}$ inhomogeneities decrease the critical temperature of the magnetic ordering. This our conclusion agrees qualitatively with the data of $[9,10]$.

Summarizing, in this work we have studied how nonmagnetic dopants, which change the local coupling of spins to the host, affect low-temperature thermodynamic characteristics of Q1D quantum spin antiferromagnets. The results of our theory qualitatively and, in some cases, quantitatively describe the behavior of the magnetic susceptibility and specific heat of the Q1D system $\mathrm{BaCu}_{2}\left(\mathrm{Si}_{1-x} \mathrm{Ge}_{x}\right)_{2} \mathrm{O}_{7}$. We have shown that in some cases the strong disorder in the distribution of characteristics of magnetic impurities in quantum antiferromagnetic spin chains can be the cause of magnetic ordering, if such chains are weakly coupled to each other, while for homogeneous chains and chains with a weak disorder a small enough coupling between chains does not produce the magnetic ordering. For other values of the parameters, magnetic impurities can decrease the Néel temperature compared to that of the homogeneous Q1D spin system, which is the case for $\mathrm{BaCu}_{2}\left(\mathrm{Si}_{1-x} \mathrm{Ge}_{x}\right)_{2} \mathrm{O}_{7}$.

1. L.N. Bulaevsky, A.V. Zvarykina, Yu.S. Karimov, R.B. Lyubosky, and I.F. Shchegolev, Zh. Eksp. Teor. Fiz. 62, 725 (1972) [JETP 35, 384 (1972)].

2. L.C. Tippie and W.G. Clark, Phys. Rev. B23, 5846 (1981).

3. K. Ikegami, S.I. Kuroda, M. Saito, K. Saito, M. Sugi, T. Nakamura, M. Matsumoto, and Y. Kawabata, Phys. Rev. B35, R3667 (1987).

4. C. Dasgupta and S.-K. Ma, Phys. Rev. B22, 1305 (1980).

5. A. Klümper and A.A. Zvyagin, Phys. Rev. Lett. 81, 4975 (1998).

6. A.A. Zvyagin, Phys. Rev. B62, R6069 (2000).

7. A. Klümper and A.A. Zvyagin, J. Phys.: Condens. Matter 12, 8705 (2000).

8. A.A. Zvyagin, Fiz. Nizk. Temp. 28, 1274 (2002) [Low Temp. Phys. 28, 907 (2002)].

9. T. Masuda, A. Zheludev, K. Uchinokura, J.-H. Chung, and S. Park, Phys. Rev. Lett. 93, 077206 (2004).

10. T. Yamada, Z. Hiroi, and M. Takano, J. Solid State Chem. 156, 101 (2001); T. Yamada, M. Takano, and Z. Hiroi, J. Alloys Comp. 317-318, 171 (2001).

11. A.A. Zvyagin and A.V. Makarova, Fiz. Nizk. Temp. 30, 639 (2004) [Low Temp. Phys. 30, 476 (2004)].

12. A.M. Tsvelick and P.B. Wiegmann, Adv. Phys. 32, 453 (1983).

13. R.B. Griffiths, Phys. Rev. Lett. 23, 17 (1969).

14. A.A. Zvyagin and A.V. Makarova, Fiz. Nizk. Temp. 30, 1095 (2004) [Low Temp. Phys. 30, 822 (2004)].

15. M. Sato and M. Oshikawa, Phys. Rev. B69, 054406 (2004).

16. J.C. Bonner and M.E. Fisher, Phys. Rev. 135, A640 (1964).

17. I. Affleck and M. Oshikawa, Phys. Rev. B62, 9200 (2000). 